\title{
Impacto subespinhal do quadril: Resultados clínicos e radiográficos do tratamento artroscópico*
}

\section{Subspine Hip Impingement: Clinical and Radiographic Results of its Arthroscopic Treatment}

\author{
Bruno Dutra Roos ${ }^{1}$ (1) Milton Valdomiro Roos ${ }^{1}$ Antero Camisa Júnior ${ }^{1}$ \\ Ezequiel Moreno Ungaretti Lima ${ }^{1}$ Mariano Feraboli Fontana ${ }^{1}$ Roger Pires Okamoto ${ }^{1}$ \\ 1 Serviço de Cirurgia do Quadril, Hospital Ortopédico de Passo Fundo, \\ Passo Fundo, RS, Brasil \\ Rev Bras Ortop 2020;55(6):722-727. \\ Endereço para correspondência Bruno Dutra Roos, MD, Serviço de \\ Cirurgia do Quadril, Hospital Ortopédico de Passo Fundo, Rua Sete de \\ Setembro, 817, Passo Fundo, RS, 99010-121, Brasil \\ (e-mail: brunodroos@gmail.com).
}

\section{Resumo \\ Palavras-chave \\ - impacto femoroacetabular \\ - articulação do quadril \\ - artroscopia}

\section{Abstract}

Objetivo Avaliar os resultados clínicos e radiográficos assim como as complicações relacionadas a pacientes submetidos ao tratamento artroscópico do impacto subespinhal do quadril.

Métodos Foram avaliados retrospectivamente 25 pacientes (28 quadris) submetidos ao tratamento artroscópico de impacto subespinhal entre janeiro de 2012 e junho de 2018. O seguimento médio foi de 29,5 meses, e os pacientes foram avaliados clinicamente pelo Harris hip score modificado por Byrd (MHHS), o non-arthritic hip score (NAHS), e quanto à rotação interna e flexão do quadril. Além disso, foram avaliados por exames de imagem: o ângulo center-edge (CE) acetabular, o ângulo alfa, a presença de sinal da parede posterior, o grau de artrose, a presença de ossificação heterotópica do quadril e a classificação de Hetsroni para Impacto Subespinhal.

Resultados Observou-se aumento médio pós-operatório de 26,9 pontos para o MHHS, 25,4 para o NAHS ( $p<0,0001), 10,5^{\circ}$ na rotação interna $(p<0,0024)$ e $7,9^{\circ}$ para flexão do quadril $(p<0,0001)$. Quanto à avaliação radiográfica, observou-se redução média de $3,3^{\circ}$ no ângulo $C E$ e de $31,6^{\circ}$ para o ângulo alfa $(p<0,0001)$. Foram classificados 18 casos (64,3\%) como artrose grau 0 de Tönnis e 10 (35,7\%) como Tönnis 1. Dois casos $(7,1 \%)$ apresentaram ossificação grau 1 de Brooker. A maioria dos quadris ( $n=15 ; 53,6 \%)$ foi classificada como tipo II de Hetsroni et al.

Conclusão No presente estudo, os pacientes submetidos a tratamento artroscópico de impacto subespinhal apresentaram melhora nos aspectos clínicos e nos padrões radiográficos aferidos pós-operatoriamente, com seguimento médio de 29,5 meses.

Objective To evaluate the clinical and radiographic results as well as complications related to patients undergoing arthroscopic treatment of subspine hip impingement. Methods We retrospectively evaluated 25 patients (28 hips) who underwent arthroscopic treatment of subspine impingement between January 2012 and June 2018. The

Trabalho desenvolvido no Departamento de Ortopedia e Traumatologia do Hospital Ortopédico de Passo Fundo, Passo Fundo, RS, Brasil.

recebido

05 de Janeiro de 2020

aceito

15 de Abril de 2020
DOI https://doi.org/

10.1055/s-0040-1713760. ISSN 0102-3616.
Copyright $\odot 2020$ by Sociedade Brasileira License terms de Ortopedia e Traumatologia. Published by Thieme Revinter Publicações Ltda, Rio de Janeiro, Brazil 


\author{
Keywords \\ - femoroacetabular \\ impingement \\ - hip joint \\ - arthroscopy
}

mean follow-up was 29.5 months, and the patients were evaluated clinically by using the Harris hip score modified by Byrd (MHHS), the non-arthritic hip score (NAHS), and in terms of internal rotation and hip flexion. In addition, the following items were evaluated by imaging exams: the center-edge (CE) acetabular angle, the Alpha angle, the presence of a sign of the posterior wall, the degree of arthrosis, the presence of heterotopic hip ossification, and the Hetsroni classification for subspine impingement. Results There was an average postoperative increase of 26.9 points for the MHHS, 25.4 for the NAHS $(p<0.0001), 10.5^{\circ}$ in internal rotation $(p<0.0024)$, and $7.9^{\circ}$ for hip flexion $(p<0.0001)$. As for the radiographic evaluation, an average reduction of $3.3^{\circ}$ in the $C E$ angle and of $31.6^{\circ}$ for the Alpha angle $(p<0.0001)$. Eighteen cases $(64.3 \%)$ were classified as grade 0 osteoarthritis of Tönnis, and 10 (35.7\%) were classified as Tönnis grade 1 . Two cases (7.1\%) presented grade 1 ossification of Brooker. Most hips ( $n=15$, 53.6\%) were classified as type II of Hetsroni et al.

Conclusion In the present study, patients undergoing arthroscopic treatment with subspine impingement showed improvement in clinical aspects and radiographic patterns measured postoperatively, with an average follow-up of 29.5 months.

\section{Introdução}

O impacto femoroacetabular (IFA) é resultante de alterações de contato entre a cabeça e colo femoral com a borda acetabular. $^{1,2}$ Essas alterações mecânicas patológicas do quadril podem lesionar a junção condrolabial e potencialmente causar alterações osteoartríticas precoces no quadril. ${ }^{3}$

Tanto o tratamento artroscópico quanto as abordagens cirúrgicas abertas (através de luxação controlada do quadril ou abordagem anterior) são amplamente descritos na literatura para o tratamento da lesão labial e restauração do contorno da junção colo-cabeça, e resultam em melhora dos resultados clínicos em pacientes com IFA sintomático. ${ }^{4}$ Apesar da ampla aplicação e sucesso dessas técnicas para tratamento cirúrgico do IFA, um subconjunto de pacientes não apresenta melhora significativa, sugerindo que fontes não reconhecidas de impacto possam existir. O IFA extra-articular resulta do contato anormal entre as regiões extra-articulares do fêmur proximal (trocânter maior, trocânter menor, e porção extracapsular do colo femoral) com o ílio ou o ísquio. ${ }^{4,5}$

Estudos recentes ${ }^{6,7}$ demonstram que a espinha ilíaca anteroinferior (EIAI) proeminente poderia contribuir como uma causa de IFA. Nesses casos, a flexão terminal do quadril resultaria em impacto com a região caudal da proeminência da EIAI contra o colo femoral. A diminuição da dor e melhora geral da função do quadril têm sido relatadas após a ressecção da proeminência da EIAI. ${ }^{8}$

O objetivo do presente estudo foi avaliar os resultados clínicos e radiográficos assim como as complicações relativas a pacientes submetidos ao tratamento artroscópico de impacto subespinhal (ISE).

\section{Materiais e Métodos}

Foram incluídos pacientes submetidos ao tratamento artroscópico de IFA, operados entre janeiro de 2012 e outubro de 2018. Nesse período, submeteram-se a esse tratamento 275 pacientes, sendo todas as cirurgias realizadas pelo mesmo cirurgião (BDR). Os critérios de exclusão do estudo foram pacientes portadores de IFA sem a presença de impacto subespinhal (240 casos), perda de seguimento do paciente, e/ou falta de exames para as aferições radiográficas (10 casos).

Todos os pacientes foram convocados e reavaliados. Os autores do presente estudo assinaram termo de compromisso para a utilização de dados. Deve-se ressaltar ainda que o projeto de pesquisa foi aprovado pelo comitê de ética em pesquisa sob o número CAAE 08965619.0.0000.5342.

De acordo com os critérios estabelecidos, 25 pacientes preencheram todos os requisitos necessários. Desses, 18 (72\%) eram do sexo masculino e a média de idade foi de 32,1 anos (desvio padrão [DP] = 7,2, variação de 19-44 anos). O quadril direito foi operado em 17 casos $(60,7 \%)$, o esquerdo em 11 (39,3\%), e 3 casos foram tratados bilateral e simultaneamente, totalizando 28 quadris avaliados. 0 seguimento médio foi de 29,5 meses (variação de 6-82 meses).

Quanto aos aspectos clínicos, os pacientes foram avaliados pré e pós-operatoriamente de acordo com o Harris hip score modificado por $\operatorname{Byrd}^{9}$ (MHHS), non-arthritic hip score ${ }^{10}$ (NAHS), o grau de rotação interna (RI) e de flexão do quadril acometido (com o uso de goniômetro para aferição). Todos os pacientes apresentaram teste para avaliação de impacto subespinhal positivo, que consiste em dor na flexão passiva do quadril em rotação neutra. ${ }^{11}$ De acordo com o MHHS, os resultados foram estratificados em ruins (MHHS $<70$ pontos), razoáveis (MHHS 70-79 pontos), bons (MHHS 80-89 pontos), e excelentes (MHHS 90-100).

Todos os pacientes foram avaliados por meio de radiografias (incidência anteroposterior (AP) de bacia, Dunn $45^{\circ}, 11$ e pseudoperfil de Lequesne, ${ }^{12}$ Tomografia computadorizada com Reconstrução 3D (TC 3D) e ressonância nuclear magnética (RNM). Nas radiografias pré-operatórias foram aferidos o ângulo centro-borda $(\theta C E)$, o ângulo Alfa $(\theta \alpha)$ conforme descrito por Meyer na incidência Dunn $45^{\circ},{ }^{11}$ a presença do sinal do cruzamento das linhas acetabulares, ${ }^{13}$ a presença do sinal da parede posterior, e a classificação de Tönnis ${ }^{14}$ para coxartrose. Nos pacientes com a presença do sinal do 
cruzamento das linhas acetabulares foi aferido o grau de retroversão acetabular, sendo classificado como leve quando ocorria no terço superior do acetábulo, moderado no terço médio, e grave no terço inferior. ${ }^{15}$ Nas radiografias pós-operatórias foram aferidos o $\theta \mathrm{CE}$, o $\theta \alpha$ e a presença de ossificação heterotópica conforme classificação proposta por Brooker et al. $^{16}$

O impacto subespinhal foi classificado pré-operatoriamente a partir de estudos de TC 3D baseados na morfologia EIAI conforme descrito por Hetsroni et al. ${ }^{17}$ No tipo I, a extensão distal da EIAI se localiza proximal à borda acetabular; no tipo II, a proeminência óssea do ílio estende-se até o rebordo acetabular; no tipo III, o EIAI estende-se distalmente ao rebordo acetabular.

O IFA tipo came foi definido como $\theta \alpha>50^{\circ} .{ }^{18}$ O IFA tipo pincer foi definido na presença de $\theta$ CE maior ou igual a $40^{\circ}$ em radiografias AP de bacia e/ou pseudoperfil de Lequesne, ${ }^{12}$ ou na presença do sinal do cruzamento das linhas acetabulares. ${ }^{13}$ A presença da associação de sinal do cruzamento e sinal da parede posterior indicou retroversão acetabular verdadeira. A presença isolada do sinal do cruzamento indicou retroversão acetabular relativa. ${ }^{15}$

Para evitar erros inter e intra observadores, as aferições foram acompanhadas por dois cirurgiões do Grupo do Quadril. No caso de haver discordância de mais de $3^{\circ}$ nas medidas angulares, uma nova avaliação foi executada por um terceiro cirurgião e procedeu-se, então, um consenso da aferição. Considerou-se magnificação média da radiografia AP de bacia de $15 \%$, que foi quantificada nos equipamentos do serviço.

As variáveis quantitativas de interesse não passaram pelo teste de normalidade. Assim, o teste estatístico empregado para análise das variáveis pré e pós-operatórias foi o teste de Wilcoxon (não-paramétrico para amostras dependentes), com nível de significância de 0,05.

\section{Resultados}

Dos 28 quadris avaliados, 23 (82,1\%) apresentavam impacto subespinhal associado a impactos do tipo came e pincer, 4 $(14,3 \%)$ impactos subespinhais associados a impacto tipo came, e 1 (3,6\%) quadril apresentou impacto subespinhal isolado. A maioria dos quadris $(n=15,53,6 \%)$ foi classificada como tipo II de Hetsroni et al. ${ }^{17}$ Além da ressecção da EIAI, foram realizados artroscopicamente outros tratamentos que estão enumerados na - Tabela 1.

Tabela 1 Descrição dos tipos de tratamentos artroscópicos realizados

\begin{tabular}{|l|l|}
\hline Tratamento artroscópico & $\mathbf{N}^{\circ}$ de casos \\
\hline Ressecção da EIAI & 28 casos \\
\hline Osteocondroplastia femoral & 27 casos \\
\hline Osteocondroplastia acetabular & 23 casos \\
\hline Desbridamento de lábio & 16 casos \\
\hline Refixação de lábio & 6 casos \\
\hline Liberação do tendão do iloipsoas & 3 casos \\
\hline
\end{tabular}

Abreviatura: EIAI, espinha ilíaca anteroinferior.
Com relação à avaliação do escore clínico MHHS, ${ }^{9}$ observouse média pré-operatória de 62,3 pontos ( $\mathrm{DP}=6,2$, variação de 50-72 pontos) e pós-operatória de 89,2 pontos ( $\mathrm{DP}=5.7$, variação de 77-100 pontos), com aumento pós-operatório médio de 26,9 pontos. Quanto ao escore clínico NAHS, ${ }^{10}$ observou-se pontuação média pré-operatória de 65.7 pontos ( $\mathrm{DP}=8,7$, variação de $45-80$ pontos) e pós-operatória de 91,1 pontos ( $\mathrm{DP}=5,8$, variação de $80-100$ pontos), com aumento médio pós-operatório de 25,4 pontos. A aferição da RI do quadril apresentou média pré-operatória de $8,9^{\circ}\left(\mathrm{DP}=7,6^{\circ}\right.$, variação de $\left.0-30^{\circ}\right)$ e pós-operatória de $19,5^{\circ}\left(\mathrm{DP}=5,7\right.$, variação de $\left.10-30^{\circ}\right)$, com aumento pós-operatório médio de $10,5^{\circ}$. Quanto à flexão do quadril, houve média pré-operatória de $111,1^{\circ}(\mathrm{DP}=10,7$, variação de $\left.90-130^{\circ}\right)$ e pós-operatória de $118,9^{\circ}(\mathrm{DP}=6,3$, variação de $100-130^{\circ}$ ), com aumento pós-operatório médio de $7,9^{\circ}$. De acordo com os critérios estabelecidos baseados na avaliação do escore clínico MHHS, ${ }^{9}$ dos 28 quadris tratados, 16 (57,1\%) apresentaram resultados clínicos bons e 12 (42,9\%) excelentes. Observou-se diferença estatística $(p<0,005)$ nas aferições clínicas pré e pós-operatórias dos escores clínicos MHHS $^{9}$ e NAHS, ${ }^{10}$ além da RI e flexão do quadril.

Com relação à avaliação radiográfica pré-operatória, a média para o $\theta C E$ foi de $34,7^{\circ}\left(22-48^{\circ}\right)$ para o $\theta \alpha$ foi de $72^{\circ}$ $\left(48-86^{\circ}\right)$. O sinal do cruzamento das linhas acetabulares foi constatado em 23 pacientes (82,1\%), sendo todos os casos classificados com retroversão leve. 0 sinal da parede posterior foi evidenciado em 15 pacientes (53,6\%). Em 15 casos $(53,6 \%)$ foi constatada retroversão acetabular verdadeira, e em 8 , relativa (28,6\%). Foram classificados 18 casos $(64,3 \%)$ como artrose grau 0 de Tönnis e $10(35,7 \%)$ como Tönnis 1 .

Quanto ao $\theta \mathrm{CE}$ foi evidenciado pós operatoriamente redução média de $3,3^{\circ}\left(22-39^{\circ}\right)$. Para o $\theta \alpha$, houve redução média de $31,6^{\circ}\left(28-52^{\circ}\right)$ (-Tabela 2). No pós-operatório tardio não foi evidenciada ossificação heterotópica em 26 casos (92,9\%) e dois casos (7,1\%) apresentaram ossificação grau 1 de Brooker. ${ }^{16}$ Como complicações verificamos um caso $(0,28 \%)$ com pequena lesão cutânea vaginal decorrente da tração, com melhora completa em 6 semanas. (-Figuras 1-5)

Tabela 2 Comparação das medidas pré e pós-operatórias, das aferições clínicas e radiográficas

\begin{tabular}{|l|l|l|l|}
\hline \multicolumn{2}{|l|}{ Avaliação } & \multicolumn{2}{l|}{$P$} \\
\hline MHHS & Pré-operatório & 62,3 & $p<0,0001$ \\
& Pós-operatório & 89,2 & \\
\hline NAHS & Pré-operatório & 65,7 & $p<0,0001$ \\
& Pós-operatório & 91,1 & \\
\hline RI & Pré-operatório & $8,9^{\circ}$ & $p<0,0001$ \\
& Pós-operatório & $19,5^{\circ}$ & \\
\hline Flex & Pré-operatório & $111,1^{\circ}$ & $p<0,0024$ \\
& Pós-operatório & $118,9^{\circ}$ & \\
\hline$\theta \alpha$ & Pré-operatório & $72,0^{\circ}$ & $p<0,0001$ \\
& Pós-operatório & $40,3^{\circ}$ & \\
\hline$\theta C E$ & Pré-operatório & $34,7^{\circ}$ & $p<0,0001$ \\
& Pós-operatório & $31,4^{\circ}$ & \\
\hline
\end{tabular}

Abreviaturas: MHHS, Harris hip score modificado; NAHS, non-arthritic hip score; RI, rotação interna do quadril; $\theta \alpha$, ângulo Alfa; $\theta C E$, ângulo CE. 


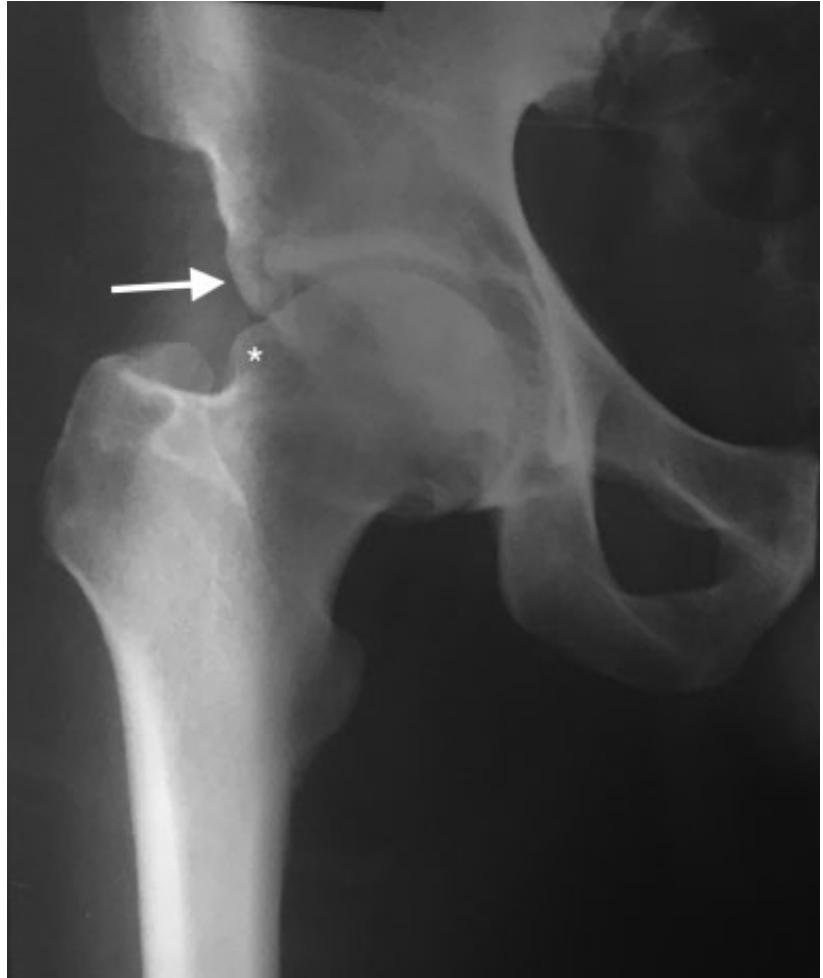

Fig. 1 Radiografia pré-operatória em incidência anteroposterior de articulação coxofemoral, evidenciando a presença de espinha ilíaca anteroinferior proeminente (seta branca) e deformidade na transição colo-cabeça (asterisco). Ângulo CE: $36^{\circ}$.

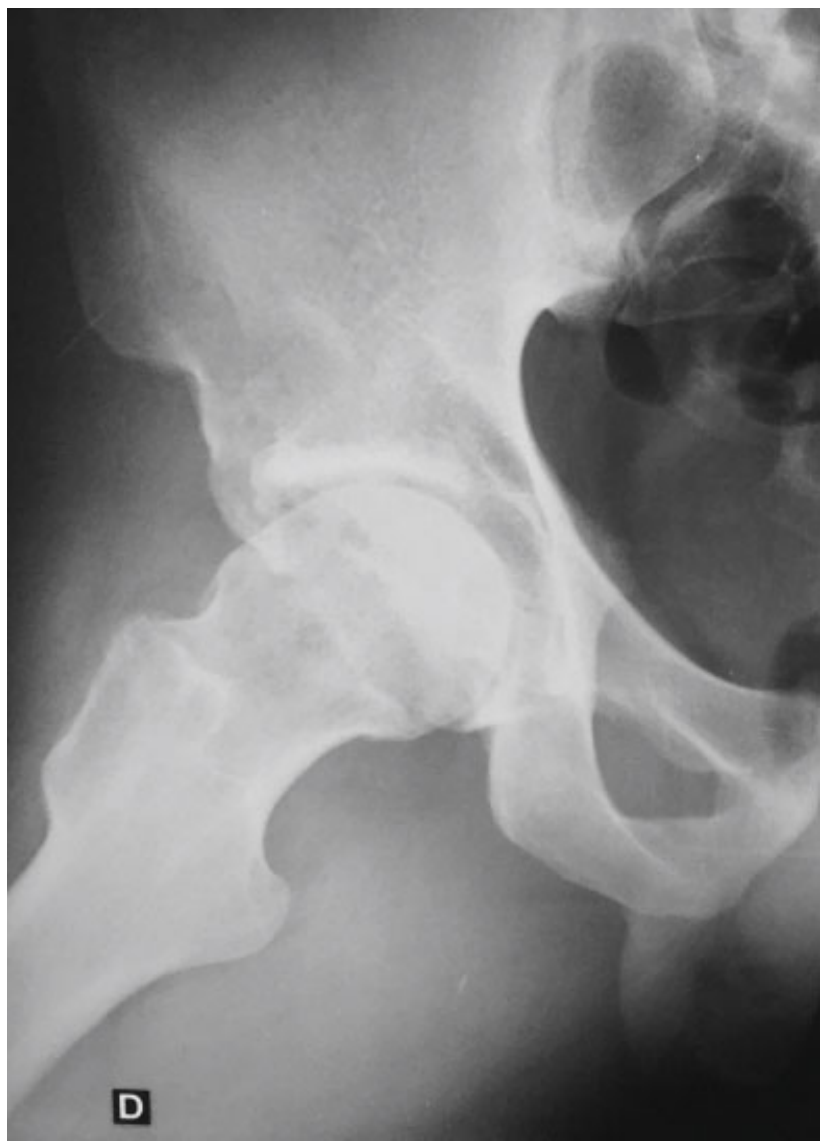

Fig. 2 Radiografia pré-operatória em incidência DUNN $45^{\circ}$ evidenciando a presença de deformidade tipo came. Ângulo alfa: $80^{\circ}$.

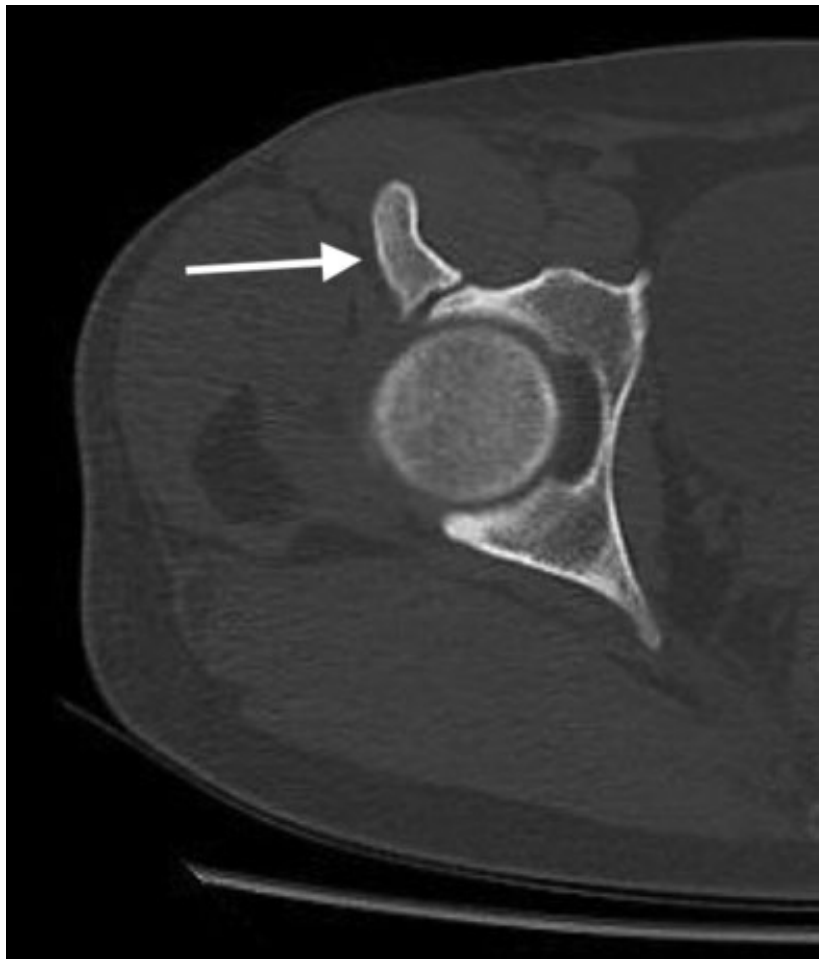

Fig. 3 Imagem pré-operatória de Tomografia computadorizada em corte axial, evidenciando a presença de espinha ilíaca anteroinferior proeminente (seta branca).

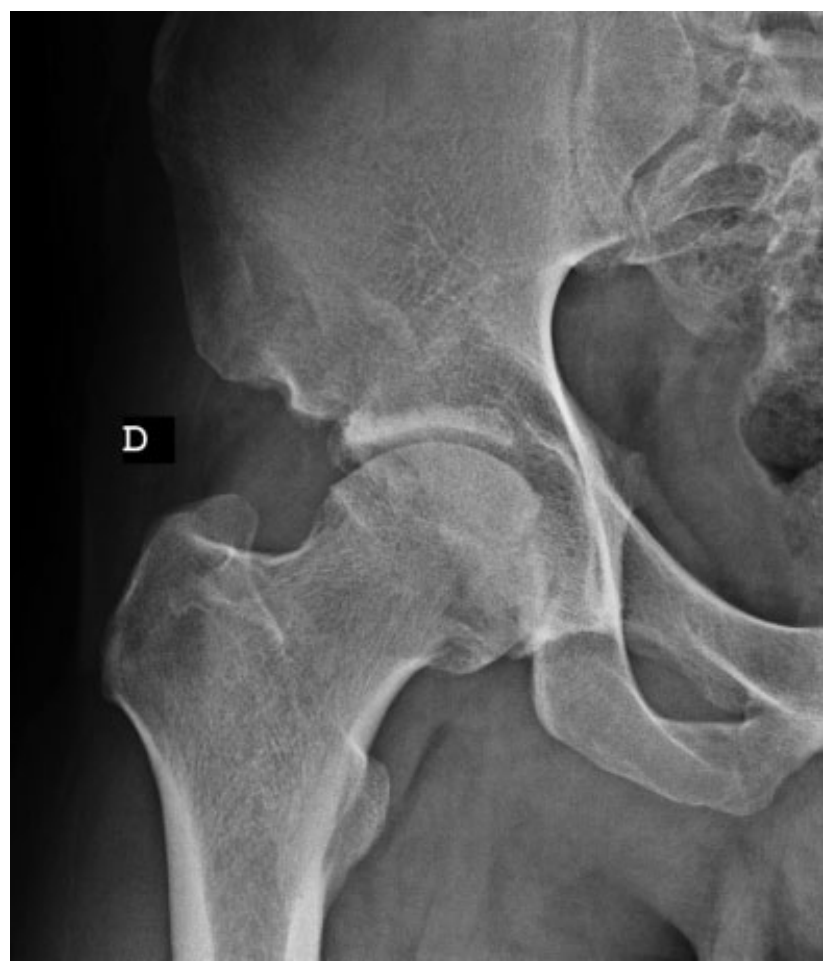

Fig. 4 Radiografia pós-operatória de 2 anos em incidência anteroposterior de articulação coxofemoral, evidenciando a correção do impacto subespinhal e da deformidade da transição colo-cabeça. 


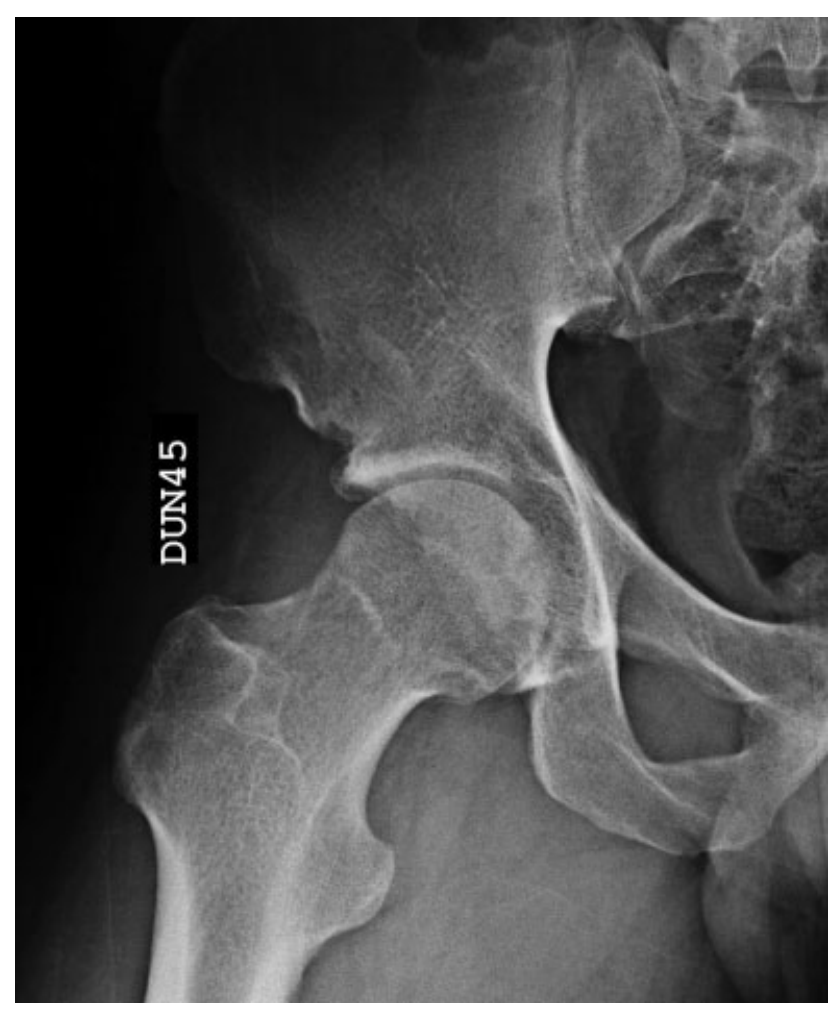

Fig. 5 Radiografia pós-operatória de dois anos em incidência DUNN $45^{\circ}$ evidenciando a correção da deformidade tipo came. Ângulo alfa: $38^{\circ}$.

\section{Discussão}

O tratamento cirúrgico do IFA é reconhecido na literatura como tratamento eficaz baseado no remodelamento do fêmur proximal e acetábulo e no tratamento das lesões condrais e labiais, a fim de diminuir o impacto do fêmur contra o rebordo acetabular e, consequente, melhorar a amplitude de movimento do quadril. ${ }^{18,19}$ No entanto, há um subgrupo de pacientes com desfechos desfavoráveis após a cirurgia sugerindo que causas não reconhecidas de impacto possam coexistir. ${ }^{20}$ Mais recentemente, trabalhos vêm sendo realizados com o propósito de identificar e compreender causas extra-articulares de impacto do quadril. ${ }^{21-23}$

O ISE é causado por uma proeminência da EIAI ocasionando um contato anormal com o colo femoral distal durante a flexão do quadril. ${ }^{21}$ A literatura demonstra que essa condição é mais comum em homens ativos, jovens (na faixa etária compreendida de 14-30 anos), e tendem a apresentar resultados mais baixos no pré-operatório para o MHHS. ${ }^{21,22}$ Frequentemente os pacientes relatam dor na face anterior do quadril ou virilha agravada pela flexão ativa do quadril.

Devido à infrequência da forma isolada de ISE, poucos trabalhos na literatura apresentam resultados do seu tratamento. Contudo, bons resultados foram demonstrados em trabalhos de séries mistas (ISE associado a formas intrarticulares de IFA). ${ }^{7,24,25}$ Nwachukwu et al. ${ }^{26}$ publicaram os resultados de 33 pacientes com ISE isolado submetidos ao tratamento artroscópico, com seguimento médio de 19 meses. Todos os pacientes eram do sexo feminino e a maioria dos quadris apresentava EIAI classificada como tipo II. Foi constatado no final do seguimento aumento médio de 22,3 pontos no MHHS. Hetsroni et al. ${ }^{6}$ realizaram revisão retrospectiva de 10 pacientes sintomáticos submetidos ao tratamento artroscópico de ISE, e relataram melhora significativa na amplitude de movimento do quadril e do MHHS.

Em nosso estudo, obtivemos resultados que estão em concordância com os descritos na literatura. Foi observada melhora clínica pós-operatória desses pacientes, com melhora dos escores de avaliação da dor, assim como aumento na amplitude de movimento do quadril (principalmente a rotação interna). Também foi constatada melhora nos padrões radiográficos aferidos. Cabe ressaltar, com base na análise radiográfica, que uma parcela expressiva dos pacientes (15 casos) apresentou sinal do cruzamento acetabular (diagnóstico de retroversão acetabular), denotando alterações na orientação das paredes acetabulares com cobertura acetabular anterior aumentada.

As limitações do presente estudo são o pequeno número de pacientes, o curto tempo de seguimento médio $(29,5$ meses), e a ausência de análise por TC pós-operatória para confirmar a adequação da ressecção da EIAI.

\section{Conclusão}

No presente estudo, os pacientes submetidos a tratamento artroscópico de impacto subespinhal apresentaram melhora nos aspectos clínicos e nos padrões radiográficos aferidos pós-operatoriamente.

Conflito de Interesses

Os autores declaram não haver conflito de interesses.

\section{Referências}

1 Ganz R, Gill TJ, Gautier E, Ganz K, Krügel N, Berlemann U. Surgical dislocation of the adult hip a technique with full access to the femoral head and acetabulum without the risk of avascular necrosis. J Bone Joint Surg Br 2001;83(08):1119-1124

2 Ito K, Minka MA II, Leunig M, Werlen S, Ganz R. Femoroacetabular impingement and the cam-effect. A MRI-based quantitative anatomical study of the femoral head-neck offset. J Bone Joint Surg Br 2001;83(02):171-176

3 Wagner S, Hofstetter W, Chiquet M, et al. Early osteoarthritic changes of human femoral head cartilage subsequent to femoroacetabular impingement. Osteoarthritis Cartilage 2003;11(07): 508-518

4 Espinosa N, Beck M, Rothenfluh DA, Ganz R, Leunig M. Treatment of femoro-acetabular impingement: preliminary results of labral refixation. Surgical technique. J Bone Joint Surg Am 2007;89 (Suppl 2 Pt.1):36-53

5 Ali AM, Teh J, Whitwell D, Ostlere S. Ischiofemoral impingement: a retrospective analysis of cases in a specialist orthopaedic centre over a four-year period. Hip Int 2013;23(03):263-268

6 Hetsroni I, Larson CM, Dela Torre K, Zbeda RM, Magennis E, Kelly BT. Anterior inferior iliac spine deformity as an extra-articular source for hip impingement: a series of 10 patients treated with arthroscopic decompression. Arthroscopy 2012;28(11):16441653

7 Larson CM, Kelly BT, Stone RM. Making a case for anterior inferior iliac spine/subspine hip impingement: three representative case reports and proposed concept. Arthroscopy 2011;27(12):1732-1737

8 Pan H, Kawanabe K, Akiyama H, Goto K, Onishi E, Nakamura T. Operative treatment of hip impingement caused by hypertrophy 
of the anterior inferior iliac spine. J Bone Joint Surg Br 2008;90 (05):677-679

9 Guimarães RP, Alves DPL, Azuaga TL, et al. Tradução e adaptação transcultural do Harris Hip Score modificado por Byrd. Acta Ortop Bras 2010;18(06):339-342

10 Del Castillo LN, Leporace G, Cardinot TM, Levy RA, Oliveira LP. Translation, cross-cultural adaptation and validation of the Brazilian version of the Nonarthritic Hip Score. Sao Paulo Med J 2013; 131(04):244-251

11 Meyer DC, Beck M, Ellis T, Ganz R, Leunig M. Comparison of six radiographic projections to assess femoral head/neck asphericity. Clin Orthop Relat Res 2006;445(445):181-185

12 Clohisy JC, Carlisle JC, Beaulé PE, et al. A systematic approach to the plain radiographic evaluation of the young adult hip. J Bone Joint Surg Am 2008;90(Suppl 4):47-66

13 Li PL, Ganz R. Morphologic features of congenital acetabular dysplasia: one in six is retroverted. Clin Orthop Relat Res 2003; (416):245-253

14 Busse J, Gasteiger W, Tönnis D. [A new method for roentgenologic evaluation of the hip joint-the hip factor]. Arch Orthop Unfallchir 1972;72(01):1-9

15 Jamali AA, Mladenov K, Meyer DC, et al. Anteroposterior pelvic radiographs to assess acetabular retroversion: high validity of the “cross-over-sign”. J Orthop Res 2007;25(06):758-765

16 Brooker AF, Bowerman JW, Robinson RA, Riley LH Jr. Ectopic ossification following total hip replacement. Incidence and a method of classification. J Bone Joint Surg Am 1973;55(08): 1629-1632

17 Hetsroni I, Poultsides L, Bedi A, Larson CM, Kelly BT. Anterior inferior iliac spine morphology correlates with hip range of motion: a classification system and dynamic model. Clin Orthop Relat Res 2013;471(08):2497-2503
18 Matsuda DK, Carlisle JC, Arthurs SC, Wierks CH, Philippon MJ. Comparative systematic review of the open dislocation, miniopen, and arthroscopic surgeries for femoroacetabular impingement. Arthroscopy 2011;27(02):252-269

19 Byrd JW, Jones KS. Arthroscopic management of femoroacetabular impingement: minimum 2-year follow-up. Arthroscopy 2011; 27(10):1379-1388

20 Ricciardi BF, Fabricant PD, Fields KG, Poultsides L, Zaltz I, Sink EL. What are the demographic and radiographic characteristics of patients with symptomatic extraarticular femoroacetabular impingement? Clin Orthop Relat Res 2015;473(04):1299-1308

21 de Sa D, Alradwan H, Cargnelli S, et al. Extra-articular hip impingement: a systematic review examining operative treatment of psoas, subspine, ischiofemoral, and greater trochanteric/pelvic impingement. Arthroscopy 2014;30(08):1026-1041

22 Cvetanovich GL, Harris JD, Erickson BJ, Bach BR Jr, Bush-Joseph CA, Nho SJ. Revision hip arthroscopy: a systematic review of diagnoses, operative findings, and outcomes. Arthroscopy 2015;31 (07):1382-1390

23 Sutter R, Pfirrmann CWA. Atypical hip impingement. AJR Am J Roentgenol 2013;201(03):W437-42

24 Hapa O, Bedi A, Gursan O, et al. Anatomic footprint of the direct head of the rectus femoris origin: cadaveric study and clinical series of hips after arthroscopic anterior inferior iliac spine/ subspine decompression. Arthroscopy 2013;29(12):1932-1940

25 Matsuda DK, Calipusan CP. Adolescent femoroacetabular impingement from malunion of the anteroinferior iliac spine apophysis treated with arthroscopic spinoplasty. Orthopedics 2012;35(03): e460-e463

26 Nwachukwu BU, Chang B, Fields K, et al. Outcomes for Arthroscopic Treatment of Anterior Inferior Iliac Spine (Subspine) Hip Impingement. Orthop J Sports Med 2017;5(08):2325967117723109 